\title{
EDITORIAL
}

\section{Improving outcomes from community-acquired pneumonia: we need to be more sophisticated about cause and effect}

\author{
G.W. Waterer* and D. Lopez ${ }^{\#}$
}

(N) uality of care indicators are being introduced in many countries for many diseases, in an attempt by the funders of healthcare to ensure optimal outcomes are being achieved. However, the extent to which an improvement in performance, in one or more of these indicators, actually improves the outcomes of patients with community-acquired pneumonia (CAP) remains very unclear.

In the current issue of the European Respiratory Journal, MENÉNDEZ et al. [1] publish their analysis of the relationship between various measures of quality of care in patients with CAP and clinical outcomes in a large multicentre Spanish study. The three quality measures they focused on were: adherence to antibiotic guidelines, delivery of the first dose of antibiotics within $6 \mathrm{~h}$ of presentation, and measurement of oxygenation at presentation. Consistent with the findings of other groups, adherence to antibiotic guidelines was associated with a shorter length of stay in patients without organ failure. In patients with more severe sepsis, mortality was also better in those who received guideline-concordant antibiotics within the first $6 \mathrm{~h}$, but not in those who received them at a later time point. Measurement of oxygenation did not predict clinical outcome, although overall adherence to this measure was $90 \%$.

The problem with almost all the literature looking at quality of care indicators in CAP, including the study by MENÉNDEZ et al. [1], is the failure to address and identify preventable mortality. Distinguishing between preventable and inevitable mortality is very important because when detailed analyses of the causes of death from CAP were performed in the USA [2] and the UK [3] they did not identify any potential for improvements in management to alter the outcome. Since nearly half of all deaths in patients with CAP are generally thought to be due to factors other than primary sepsis [4], it is critically important that we understand the cause and effect relationship between physician behaviour (such as guideline compliance or rapid antibiotic administration) and patient outcome.

If we look at compliance with antibiotic guidelines, it is easy to see how a regime that failed to cover common pathogens

\footnotetext{
*School of Medicine and Pharmacology and Lung Institute of Western Australia, University of Western Australia, Perth, Australia. " Dept of Critical Care Medicine, Fundaciòn Jiménez Diaz-Capio University Hospital, Madrid, Spain.

CORRESPONDENCE: G. Waterer, School of Medicine and Pharmacology and Lung Institute of Western Australia, University of Western Australia, Level 4 MRF Building, Royal Perth Hospital, Wellington St, Perth 6000, Australia, E-mail grant.waterer@uwa.edu.au
}

(guideline minus) could result in adverse outcomes. However, MENÉNDEZ et al. [1] do not present any data to suggest that key pathogens were not covered. Just as common, if not more so, in descriptions of antibiotic use is physicians adding antibiotics to guideline-recommended regimes (guideline plus). In this case, unless there is clear evidence of antibiotic-mediated toxicity, it is difficult to see how this would directly impact on outcome. More probable is that the treating physician is concerned about multi-resistant or unusual pathogens because of some facet of the presentation or comorbidities not adequately captured or accounted for by multivariate analysis.

Is there any harm in having quality of care measures? Certainly there is some evidence in the USA that the push to give antibiotics in shorter time frames has led to their overuse [5, 6]. More importantly though, is that fixation on these measures may hinder real progress. For example, if all 10-yr-olds sit a standard mathematics test, analysis might show that students who take between 90 and $120 \mathrm{~min}$ to complete the test achieve the best results. Using the same argument as the time to antibiotics quality measure, the approach to improving mathematic test results in 10 -yr-olds would be to reward schools based on how many students finish the test in less than $120 \mathrm{~min}$. In the same way as this approach ignores the complex reasons behind why students who take longer get worse test results, focusing purely on how quickly antibiotics are administered also ignores the complex nature of optimal medical management of patients with CAP.

Clearly, taking $8 \mathrm{~h}$ to give antibiotics does not represent as good a quality of health care delivery as giving them within $2 \mathrm{~h}$. However, early recognition of type II respiratory failure and institution of noninvasive ventilation to avoid intubation; appropriate fluid resuscitation to avoid pre-renal renal failure; tight control of blood glucose; prophylaxis for thrombotic events; and correction of electrolyte disturbances that may predispose to arrhythmia and a host of other factors may be far more important in determining outcome. Similarly, for length of hospital stay, the frequency with which patients are reviewed and the level of experience of the treating clinician may be critical factors [7].

Is it possible that physicians who use guideline-compliant antibiotic therapy and hospital systems that can see, diagnose and treat patients faster, are more likely to pay attention to all the above facets of medical care? If a hospital or a physician changes practice to enforce guideline-compliant antibiotic therapy within $6 \mathrm{~h}$ but then doesn't attend to all the other medical issues (and 
perhaps doesn't then review the patient for 24-36 h), do we really think the outcomes will improve?

Despite its limitations the study by MENÉNDEZ et al. [1] is important because it again demonstrates that there are differences in patient outcomes not simply explained by comorbidities or severity at presentation, but due to differences in medical management. The challenge now is to define the bundle of practices that result in best patient outcomes, much like that being developed with the surviving sepsis campaign $[8,9]$, and truly identify patients who have adverse outcomes that are preventable.

\section{STATEMENT OF INTEREST}

None declared.

\section{REFERENCES}

1 Menéndez R, Torres A, Reyes S, et al. Initial management of pneumonia and sepsis: factors associated with improved outcome. Eur Respir J 2012; 39: 156-162.

2 Marrie TJ. Deaths in risk classes I-III: a measure of quality of care in patients hospitalised with CAP? Eur Respir J 2004; 23: 103-105.
3 Simpson JC, Macfarlane JT, Watson J, et al. A national confidential enquiry into community acquired pneumonia deaths in young adults in England and Wales. British Thoracic Society Research Committee and Public Health Laboratory Service. Thorax 2000; 55: 1040-1045.

4 Mortensen EM, Coley CM, Singer DE, et al. Causes of death for patients with community-acquired pneumonia: results from the Pneumonia Patient Outcomes Research Team cohort study. Arch Intern Med 2002; 162: 1059-1064.

5 Welker JA, Huston M, McCue JD. Antibiotic timing and errors in diagnosing pneumonia. Arch Intern Med 2008; 168: 351-356.

6 Drake DE, Cohen A, Cohn J. National hospital antibiotic timing measures for pneumonia and antibiotic overuse. Qual Manag Health Care 2007; 16: 113-122.

7 Bewick T, Cooper VJ, Lim WS. Does early review by a respiratory physician lead to a shorter length of stay for patients with nonsevere community-acquired pneumonia? Thorax 2009; 64: 709-712.

8 Coba V, Whitmill M, Mooney R, et al. Resuscitation bundle compliance in severe sepsis and septic shock: improves survival, is better late than never. J Intensive Care Med 2011; 26: 304-313.

9 Daniels R, Nutbeam T, McNamara G, et al. The sepsis six and the severe sepsis resuscitation bundle: a prospective observational cohort study. Emerg Med J 2011; 28: 507-512. 